\title{
User Testing of a Scottish Intercollegiate Guideline Network Public Guideline for the Parents of Children with Autism.
}

Naomi Fearns ( $\square$ naomi.fearns2@nhs.scot)

Healthcare Improvement Scotland

Laura Walker

Healthcare Improvement Scotland

Karen Graham

Healthcare Improvement Scotland

Norman Gibb

Healthcare Improvement Scotland

Duncan Service

Healthcare Improvement Scotland

\section{Research Article}

Keywords: Guidelines, Clinical practice guideline, Patient guidelines, Patient version, Public version, Autism, User testing

Posted Date: August 19th, 2021

DOl: https://doi.org/10.21203/rs.3.rs-783695/v1

License: (9) (1) This work is licensed under a Creative Commons Attribution 4.0 International License.

Read Full License

Version of Record: A version of this preprint was published at BMC Health Services Research on January 15th, 2022. See the published version at https://doi.org/10.1186/s12913-021-07384-2. 


\section{Abstract}

Background: The Scottish Intercollegiate Guidelines Network (SIGN) is the leading national clinical guideline producer in Scotland. Improved design and dissemination of guidelines produced for the public can empower people to take an active role in self-management and shared decision-making. The aim of this study was to test a public version of a guideline with the parents of children and young people with autism, implement improvements, and identify what works in making it usable and accessible.

Methods: Parents were recruited from across Scotland. User testing involved a formal 'think aloud' process and semi-structured interview that guided users through the booklet. Sessions took place individually and were recorded and transcribed. Key findings were identified and themed using the honeycomb user experience model.

Results: Fourteen user-testing sessions were conducted. Key facilitators for usability and desirability of the guideline included the chunking of text, consistent use of colour and the use of boxes to highlight important information. Simple language, written in a tone of partnership, helped to engage parents. Value arose from the guidelines ability to explain the process of diagnosis and make parents feel empowered in their relationship with healthcare professionals. There was a lack of consensus on the usefulness of rating the strength of evidence and recommendations.

Conclusion: There was a marked similarity between what was important to the parents and what is important for other groups. The involvement of patients and carers in the guidelines development was key to its credibility. One size does not fit all in presenting evidence-based recommendations to the public and it is a challenge to provide sufficient information while avoiding information overload.

Recommendations and evidence levels are suitable for use in public versions, but these should be kept as simple as possible.

\section{Background}

Clinical guidelines are tools that provide evidence based recommendations and direct appropriate healthcare [1-3]. Traditionally guidelines are developed to guide clinicians' decision-making, but there is a growing interest and demand for public versions of clinical guidelines. In the UK, both the Scottish Intercollegiate Guideline Network (SIGN) and the National Institute for Health and Care Excellence (NICE) produce versions of their guidelines for the public. Public versions can make patients feel empowered to participate more actively in their care and engage in shared decision-making [4]. Despite the growing recognition of the value of public versions, an evaluation of guidelines on the resection of hepatocellular carcinoma found that only 2 of the 22 guidelines had developed information for the public [5] and a review found just $24 \%$ of tools developed to aid the implementation of guidelines were for the public [6].

The DECIDE (Developing and Evaluating Communication Strategies to support Informed Decision and practice based on Evidence) project [7] found that awareness of clinical guidelines is low in the general public, and that even when people are aware of them they may not perceive them any more positively 
than alternative sources of health information $[1,4,8]$. The project concluded that members of the public want actionable information to help them choose between treatments and that help them participate in shared decision-making. "One size does not fit all" in public versions, but it is clear that a guideline must be attractive, easy to use and be available in multiple formats to be accessible and useful to the public [7].

User testing has been used successfully to evaluate and improve systematic reviews, guideline creation tools [9] and dissemination methods with healthcare professionals [10-12] and policy makers [13], as well as the public [8]. User testing identifies fixable flaws in guideline design, which make the guideline inaccessible if they are not corrected [8]. Many questions still remain about how best to format and disseminate guidelines to different groups [9]. Continued user testing of SIGN public versions will ensure that they are accessible and inform the growing evidence base in this area. The aim of this study was to test and refine a SIGN public version for the parents of children and young people with autism. It also sought to explore how transferable the previous findings of the DECIDE project $[1,4,8,14]$ were to the parents of children with autism.

\section{Methods}

\section{Participants and setting}

Participants were recruited through the SIGN patient network (a 'virtual' group of patients, carers, and members of the public), NHS Greater Glasgow and Clyde Child and Adolescent Mental Health Service and from third sector organisations including the National Autistic Society, Autism Network Scotland, and local carers' centres. Snowball sampling was also used. The SIGN research analyst (LW) contacted organisations, and potential participants were approached by email and telephone. A written information summary of the evaluation was provided to all participants prior to the user testing session. Testing took place at a location convenient to participants and travel expenses were provided. The names used in quotations are pseudonyms. We aimed to recruit 12 to 15 participants because our previous user testing suggested that this would be sufficient to reach a saturation of views.

User testing took place in November 2016 in locations across Scotland including Glasgow, Edinburgh, Perth, Dundee and Arbroath.

\section{User Testing Process}

User testing was based on a method of data collection and analysis, which was developed by Rosenbaum [10]. This method uses a think-aloud protocol and a semi-structured interview guide, which involved participants being asked to verbalise their thoughts while they read the guideline. This was audio recorded and transcribed. The interview guide was structured around the honeycomb model of user experience, which has six facets: usability, credibility, usefulness/value, desirability, accessibility and findability [15]. User testing took place individually and face-to-face and lasted approximately one hour. One interviewer (LW) conducted all the interviews, and a SIGN public partner (a volunteer who works with 
SIGN to provide a public perspective) acted as an observer and took structured notes. The observer was not available for the final three interviews. A training session was held with the observer prior to the start of data collection.

A pilot interview was held to examine the feasibility of the interview guide, and resulted in minor amendments, including the order of the questions, and the print quality of materials.

\section{The guideline}

There are three public versions of SIGN guideline 145: Assessment, diagnosis and interventions for autism spectrum disorders [16], a booklet for young people with autism; one for adults with autism; and one for parents, carers and families. The user testing examined a draft of the public version for parents, carers and families. This guideline included information on interventions. The draft was designed on the principles identified in our previous publications $[4,8]$. Following the user testing, improvements were made to the guideline.

SIGN's previous public versions used three pre-defined levels of evidence, to explain the evidence quality underlying our advice to the public. We tested the use of an additional level of evidence, for a strong recommendation based on good quality evidence, in this booklet (see Fig. 3). The new format matches more closely with the evidence levels used in SIGN's clinical guidelines [17]. SIGN's levels of evidence are based on GRADE recommendations $[18,19]$.

SIGN public versions aim to be an accurate translation of the clinical guideline and only those interventions recommended in the guideline are included. However, further information is included to help patients and the public understand the recommendations.

\section{Analysis}

The method of analysis is described in detail in our previous paper [8]. It incorporates two phases:

- phase one identified barriers to the use of the public version and necessary amendments to the guideline and was published on SIGNs website [20],

- phase two identified issues that may be generalisable beyond this specific guideline, and is reported on here.

In phase two, the findings were refined and analysed thematically [21] using the honeycomb user experience model [15] as a framework. Two researchers (LW and NF) carried out the thematic analysis for phase two, using NVivo ${ }^{\circledR} 10$. One researcher $(L W)$ analysed the entire data set and a second researcher (NF) independently coded a purposive sample of three transcripts from different regions of Scotland. The researchers met to discuss the results and agreement was reached on key themes and findings.

\section{Ethics}

This project is classified as a NHS service evaluation. Information on the planned data collection was submitted to the East of Scotland Research Ethics Committee, who confirmed that full ethical approval 
was not required. Participants were sent an information sheet in advance, and gave informed written consent before the session began. Participants were informed of their right to withdraw at any point of the study, all data was held securely and no personally identifiable information or quotes have been published.

\section{Results}

\section{Participants}

Fourteen parents of people with autism took part in the study. Sixteen people agreed to take part but two people were unable to make the available dates. All of the participants were female, with a mean age of 42 and a range of 10 years, they were largely well educated, and involved in a range of activities (such as volunteering) in relation to autism, see table 1.

Table 1

participant information

\begin{tabular}{llll}
$\begin{array}{l}\text { Participant } \\
\text { pseudonym }\end{array}$ & Gender & Level of education & Source recruited from \\
\hline Jennifer & Female & In college level further education & SIGN patient network \\
\hline Ruth & Female & Secondary school qualifications & Snowballing \\
\hline Susan & Female & Secondary school qualifications & Carers Centre \\
\hline Lisa & Female & Undergraduate degree & SIGN patient network \\
\hline Angela & Female & Postgraduate degree & CAMHS \\
\hline Diane & Female & Higher National Diploma (HND) & Carers Centre \\
\hline Carol & Female & Postgraduate degree & Snowballing \\
\hline Karen & Female & Undergraduate degree & SIGN Facebook \\
\hline Michelle & Female & Secondary school qualifications & Snowballing \\
\hline Patricia & Female & In college level further education & SIGN patient network \\
\hline Jill & Female & Undergraduate degree & SIGN Facebook \\
\hline Rachel & Female & Undergraduate degree & Carers Centre \\
\hline Mary & Female & Higher degree & SIGN Facebook \\
\hline Heather & Female & Postgraduate degree & SIGN patient network
\end{tabular}




\section{Findings}

\section{Usefulness and value}

There was agreement from most parents that the guideline would be a valuable resource for families of children with autism, particularly at the time of assessment and diagnosis. The parts of the guideline that parents found most useful included, information on their child's health condition, tools and tests used for assessment, information on interventions, what to expect from healthcare professionals and services at each stage of diagnosis, and signposting to sources of information and support.

Parents suggested that the public version empowered them to ask informed question of healthcare professionals and to participate in shared decision-making. Information was valued, even if it was not linked to a recommendation, because it could be used in discussions with healthcare professionals and to highlight specific services/treatments (see Fig. 1). This type of information most useful when it contained 'tick boxes', or other interactive tools.

"... it [Fig. 1] tells you what the professional should be doing... I would probably refer back and say "well, I don't think we've discussed $X, Y$ or $Z$, so can you give me some more information about that"... [it] is a daunting process, for anyone, so the more information you can have the better."

- Rachel

The 'Where can you find out more? section provided a list of trusted sources of help and information. Parents wanted local support information, but this could not be provided in a national guideline. Signposting to national and third sector organisations may be the best way that a national body can meet this need.

\section{Usability.}

The number of text boxes (see Fig. 2) used in this version, sometimes three or more on a single page was confusing for parents. Chunking [22] via a mixture of simple text, bullet points and boxes or icons on each page breaks up the text in a more usable way.

Parents highlighted the importance of the consistent and meaningful use of colour. In SIGN public versions, recommendations are green, which is associated with safety or 'go'. Information boxes are highlighted in blue (see Fig. 1), which parents linked with information road signs. Inconsistencies in the use of colour, such as too many coloured boxes on one page, or text that seemed unrelated appearing in the same colour boxes, was overwhelming for some parents.

SIGN uses a system of icons with text to flag recommendations and their evidence level (see Fig. 3). Parents found the use of thumbs up, tick and question mark symbols clear and easy to understand. 
However, the response to the underlying four levels of evidence was mixed. Some parents appreciated the level of detail offered by the grades, and others thought it would be sufficient simply to know that SIGN recommended an intervention.

"I think, I suppose it's saying 'its ok', - based on research, based on clinical. I would say probably your Joe Public will just think it's one in the same thing, they're not going to be differentiating it at all."

-Diane

While the parents understood the essential message of the evidence levels, that one intervention is strongly recommended and another one less strongly recommended, most did not understand why it is necessary to have these different levels of recommendation. Similarly, parents found the use of the not enough evidence icon (see Fig. 3) disconcerting. While they understood that the question mark and text was meant to convey uncertainty, they did not like this message, or understand why guideline producers would need to use it.

...to the lay person.... What makes one good quality you know if it's basing it on evidence surely it should be of a certain quality otherwise why would you use that recommendation... surely all research should be good quality and if not why not? - Michelle

\section{Credibility.}

Parents valued the public version because they saw it as a trusted and credible source of information. Its credibility arose from the simple and clear description of the process used in the development of the guideline (See Fig. 4) and the evidence-based nature of the recommendations.

"... read it [Fig. 4] and know that that's how the process has been gone through and the information that is there has been, like accredited, if you like and you'd feel confident that it had gone through all the relevant stages to know that that's the most up-to-date information to use." - Rachel

The simple information provided on the process of guideline development reassured the parents that the public version was based on up-to-date evidence. While some people would have preferred more detailed information, this was sufficient for the majority of parents, and considered too much information by some.

Patient and carer input in the guideline development process was key to the relevance and credibility of the recommendations. Patients and carers are involved in SIGN's guideline development process, including representation on the guideline development group, and this is mentioned in the information provided in the public version. However, this information was not salient enough for all the parents and needs to be made more detailed and prominent. 
For some of the parents, it would have been reassuring if a larger group of carers and people with autism had been even more actively involved in the guideline development process to ensure that the included information was not too medical in orientation and was meaningful and actionable for carers. In the quote below, Jill explains why the more active involvement of a group of parents in the recommendation development process would be reassuring.

I kind of glossed over that [key to levels of recommendation/evidence] to be honest... Ok so there are four different types of recommendation, and it's SIGNs recommendations. Yeah, it's clear, but I don't really know who SIGN is so I don't know if I trust their opinion. Whereas if you had a body of parents and carers... who have given their thumbs up, double thumbs up, question mark or 'dubious about that, cause that's what the doctors say but it's nonsense in real life' that would probably hold more weight for me personally...

- Jill

Concerns regarding the status or enforceability of the guideline were evident. Parents wanted to be able to use it to discuss their options with healthcare professionals and to make a case for receiving the recommended interventions. Therefore they wanted to know if the guidelines were enforceable and what they could do if their children's' care was not in line with the recommendations.

...I see all the recommendations, and realise that actually, it doesn't happen... if you read this, and this isn't happening, what can you do?...because [it is] all very well having the guidelines, but if they're not being adhered to where does that leave you as a parent, can you just wander down and go 'here, I've got these guidelines' and they can say well actually I don't have to do that....

-Michelle

\section{Desirability.}

The quality of the design and presentation of the material is key to making any public version desirable. For the parents of children with autism, the use of inclusive and sensitive language and terminology was especially important. The tone should be collaborative, positive and reassuring; however, this must be balanced by the need to convey information clearly, some of which may be perceived as negative or 'scary'.

... a lot of the language I like, because in many things they talk very much about autism as 'they' as if they're some sort of strange distant individual. It seems much more collaborative... especially the bit where it talks about occupational therapist that they're people who can 'help' I think that's very good language, because sometimes it's the word "treat"...

-Karen 
Terminology can pose a significant barrier to the desirability and the credibility of a public version. Terms used to describe people with autism, provoked a strong emotional response. Parents preferred terms that that they recognised, from their child's diagnosis, from patient information sites or social media pages for carers. They had different preferences such as 'autistic person/child', 'with autism' or 'having autism'. Another example of emotive language was the term anti-psychotic, used in a recommendation about medication. Additional contextual information, which acknowledges some of these issues, might help to make this recommendation less off-putting.

... a lot of people see the word anti-psychotic and go 'why are you giving my child antipsychotics, they're not psychotic, what's going on'... a little bit more background as to it's a particular kind of medicine that is used for certain behaviours and don't let the word scare you... you know so that's a big vague...

- Mary

The inclusion of quotes from parents helped to personalise the material and make the information less medical in tone. Parents felt they could relate to the quotes, and that they helped to normalise their fears around diagnosis. Quotes also posed a potential risk to the desirability and accessibility of the booklet because what may personalise the material for one person may feel alienating to another. Parents of girls felt excluded by the majority of quotes referring to boys names. Some parents liked the supportive or emotional tone of the the quotes while others would have appreciated a more practical orientation to them.

.... I think the ones on page 5, as I said, about gender when you see two quotes, both of boys again you think "oh here we go"... maybe just a few quotes about how you would get through the medical process or "yes the waiting times are long, but ..." that sort of thing. I think a lot of the quotes are sort of emotional quotes as opposed to maybe practical quotes. - Karen

\section{Accessibility and findability.}

Parents highlighted the need to have the public versions available in multiple formats to maximise accessibility and findability. This includes, as a minimum, availability online and as hard copies.

Availability online helps people to find the guideline and make sharing the booklet with peers easier. Making hard copies of it available makes it accessible to people who do not routinely have access to the internet, and allows distribution directly by healthcare professionals, which was the strongest preference for distribution expressed by the parents.

...it would be even more appealing if the practitioners...introduced it to the family...it could be available in for example things like that first developmental assessment... because you do go away with nothing generally. And then the risk is a lot of people resort to doing Google searches and getting all sorts of stuff. - Angela 
A potential barrier to parents finding and using this public version was confusion about its exact purpose. The SIGN logo and accompanying text saying 'Scottish guidelines' was printed on the front cover and more information on who the booklet is designed for was given on page 2. Despite this, it was not clear to all of the parents that this was a version of a clinical guideline that had been designed specifically for parents and carers of children with autism. This can lead to confusion about why this booklet would be helpful for them and how and when they would be able to access it.

\section{Implementing the user testing findings}

Many of the suggestions made by parents led to changes to the final version of the booklet. For example, the font size was increased on the banner on the front page; the title was amended to include the word 'families'; and a sentence thanking parents and carers that contributed to the consultation of the booklet was moved to the inside cover to make their involvement salient. The final version of this guideline, which incorporated the results of this user testing is available on SIGN's website [23].

\section{Discussion}

The points of key importance about the credibility, usability, findability and desirability of this public versions of a clinical guideline for parents and carers of children and young people with autism, were similar to those identified by the DECIDE project in other groups $[4,8]$. These included the chunking of text [22], consistent use of colour, the use of boxes to highlight important information and simple language using a collaborative tone. Some issues and preferences about the design of the public version were more prominent in the parents of children with autism.

Consistent with previous research $[4,8,24]$ the usefulness of the public version was dependant on providing credible, evidence-based information, that parents felt empowered them to navigate the healthcare system and get involved in shared decision-making. A substantial amount of contextual information, not directly linked to the recommendations, may be required to facilitate this, and guideline makers must strike a balance between including all potentially useful information and brevity. At 58 pages the length of the booklet [23] is likely to be off-putting for some members of the public $[4,8]$. This is may be a larger problem in a less well-educated group.

There is a lack of public access to clearly trustworthy information about the effects of health interventions online[25]. Despite this lack, awareness of clinical guidelines and the availability of public versions of clinical guidelines is low in the general public, and evidence producers websites may not be easily found or user friendly $[1,4,10]$. Simple publication on guideline makers' websites is unlikely to be sufficient to disseminate information to the public [24].The preference of the public seems to be for distribution directly by healthcare professionals, however it is also important that public versions are accessible online $[4,8]$. Guideline makers should consider options, such as forming partnerships with third sector organisations who may aid dissemination by linking to public versions of relevant guidelines, or making use of other relevant portals [24]. 
Variation in the public's appetite for detail about the evidence that underlies recommendations is a difficult challenge to overcome, although layering of information in electronic resources is helpful [26]. A lack of awareness about the existence of lower quality research, and why a guideline producer would need to use it, leads to some members of the public not valuing (or understanding) the need for different levels of recommendation. When involving the public in guideline development, training can be offered to overcome this [27], however it presents a much more challenging problem for the development of public versions of guidelines. Much material created for the public based on clinical guidelines sidesteps this problem by not referring directly to levels of evidence or recommendations [27].

Work in the field is ongoing on developing quality criteria for public versions of clinical guidelines and other decision aids [28-30], and this research makes clear the importance of ensuring that public versions reflect the relevant recommendations and that recommendations are clearly flagged and not lost within an excess of other information. The GIN public toolkit [27] supports the inclusion of recommendation and evidence grades within public versions, provided that they are used alongside intuitively understandable icons (like the SIGN ticks and thumbs up symbols).

On balance, our user testing supported the use of multiple levels of recommendation. This group understood the meaning of the hierarchy of strength. However, recommendation levels must be kept as simple as possible and it should be clear how people could apply the recommendation to their healthcare context. It may also be helpful to be flexible in the application of graded recommendations and evidence levels and use a simpler approach, when making public versions for patient groups that may have lower reading ages, lower levels of health literacy [31, 32], or shorter attention spans. Where resources allow, multiple formats of public versions should be made available $[4,27]$.

Interestingly the parents of children with autism placed a greater emphasis on the active inclusion of the public in developing the clinical guideline and on the inclusion of up-to-date evidence than patients with glaucoma [8]. Parents wanted to know that the recommendations would be considered practical and useful by carers, whereas the people with glaucoma placed a stronger emphasis on the knowledge of the healthcare professionals involved in the guideline development. This may be related to the age of the two groups, the glaucoma group consisted largely of older adults who tend to prefer a more directive approach to shared decision-making [33]. Stigma associated with the diagnosis of autism [34], and the role of the parents in caring and advocating for their children may also have contributed to this preference. Many of the participants were active in the autistic community and used social media sites and other methods to connect with other parents and strongly valued the views and resources shared by this community. Community engagement strategies are one methods of making guidelines more patient centred and may be particularly useful for groups such as parents of children with autism[35].

Unsurprisingly the use of a tone of partnership and collaboration in the public version was important for these parents. Guideline developers should tailor the language used in public versions to the specified user group as far as possible, and using user testing or some other form of consultation is certainly helpful. Acknowledging any challenges with terminology and describing how chosen terms have been 
selected in the public version, may also reassure the user group that the guideline maker is aware of the sensitivity and controversy that may be associated with them.

SIGN uses consultation with patients and the public to select their recommendations for inclusion in their public versions; however, the public may still struggle to understand how to make practical use of all recommendations included. For example, recommendations that use the question mark symbol to reflect uncertainty in the evidence base. The inclusion of qualitative evidence examining patient perspectives in the guideline development process may help to make recommendations more feasible, acceptable and actionable.[36, 37]. SIGN have begun to explore how they can incorporate qualitative research more actively into their guidelines, in SIGN 159: Epilepsy in children and young people - diagnosis and management (still in development)[38] and with the recent publication of their first rapid qualitative synthesis, carried out to inform a guideline on foetal alcohol syndrome [39].

\section{Strengths and Limitations:}

SIGN's public versions of clinical guidelines have already been redesigned based on the work of the DECIDE project between 2011 and 2015 [7]. This study built on our previous user testing by using a guideline that incorporated recommendations about interventions and was targeted at a different user group to those previously studied. This allowed us to explore how transferable our previous findings were to the parents of children and young people with autism

The sample was limited to women, all of whom were mothers of a child with autism. The sample consisted mainly of highly educated women, many of whom were active in the autistic community and half of the sample was recruited from SIGNs patient network or via SIGNs Facebook page. This group was more aware of clinical guidelines than the general public and may have held stronger views on aspects of the management of autism than a more diverse sample would.

This guideline did not contain numerical information on the risks and benefits associated with treatment options. Future work could explore the publics' needs concerning this type of information by carrying out user testing with an appropriate SIGN guideline.

\section{Conclusion}

Many aspects of usability, usefulness, desirability and credibility were the same for the parents of children with autism as for other public groups [8] [4]. The involvement of patients and carers in the development of the guideline and a tone of partnership in the text was particularly important to credibility for the parents. One size does not fit all in presenting evidence-based recommendations to the public and it is a challenge to provide sufficient information to make people feel empowered while avoiding information overload. Recommendation and evidence levels should be included in most public versions, but kept as simple as possible. Guideline makers should explore multiple methods of including the patient perspective in their recommendations to make them more credible to the public and feasible to implement. This may include the use of qualitative research in the guideline development process, 
community engagement strategies, as well as more common methods like public consultation and public representatives on guideline committees.

\section{Abbreviations}

DECIDE: Developing and Evaluating Communication strategies to support Informed Decision and practice based on Evidence

GIN: Guidelines International Network

NICE: National Institute of Clinical Excellence

SIGN: Scottish Intercollegiate Guidelines Network

\section{Declarations}

\section{Ethical approval and consent to participate}

This project is classified as a NHS service evaluation. Information on the planned data collection was submitted to the East of Scotland Research Ethics Committee, who confirmed that full ethical approval was not required.

\section{Consent for publication}

Not applicable

\section{Funding}

This study was carried out as part of SIGNs service development and no additional funding was acquired.

\section{Competing interests}

No conflicts of interest have been declared.

\section{Authors' contributions}

LW and NF wrote the study protocol. LW collected the data, carried out the data analysis and drafted sections of the manuscript. NF supported the planning of the study, contributed to the analysis of the data, drafted sections of the paper and wrote the final manuscript. NG was an observer and note taker at 
the user testing sessions. DS contributed to the design and coordination of the study. KG supported engagement of patients and the public in the study. All authors read and approved the final manuscript.

\section{Availability of data and materials}

The interview transcripts are not publicly available due to the authors not having obtained consent from the participants to share their interview transcripts. They are held securely by Healthcare Improvement Scotland.

\section{Acknowledgements:}

The authors would like to thank the National Autistic Society, Autism Network Scotland, Scottish Autism, Children's Health Scotland and Carers Scotland for their assistance with recruitment for this study. We would also like to thank the DECIDE work package 3 project team.

DECIDE (Developing and Evaluating Communication strategies to support Informed Decision and practice based on Evidence) was funded by the European Union Seventh Framework Programme (Fp7/20072013) under grant agreement number 258583.

\section{References}

1. Loudon K, Santesso N, Callaghan M, Thornton J, Harbour J, Graham K, Harbour R, Kunnamo I, Liira $\mathrm{H}, \mathrm{McF}$ arlane $\mathrm{E}$ et al: Patient and public attitudes to and awareness of clinical practice guidelines: a systematic review with thematic and narrative syntheses. BMC Health Services Research 2014, 14(1):321.

2. Institute of Medicine Committee on Standards for Developing Trustworthy Clinical Practice G. In: Clinical Practice Guidelines We Can Trust. edn. Edited by Graham R, Mancher M, Miller Wolman D, Greenfield S, Steinberg E. Washington (DC): National Academies Press (US) Copyright 2011 by the National Academy of Sciences. All rights reserved.; 2011.

3. Davies E: Can you trust your clinical guidelines? BMJ: British Medical Journa/ 2013, 346:f4003.

4. Fearns N, Kelly J, Callaghan M, Graham K, Loudon K, Harbour R, Santesso N, McFarlane E, Thornton $\mathrm{J}$, Treweek S: What do patients and the public know about clinical practice guidelines and what do they want from them? A qualitative study. BMC Health Serv Res 2016, 16:74.

5. Gavriilidis P, Roberts KJ, Askari A, Sutcliffe RP, Huo TL, Liu PH, Hidalgo E, Compagnon P, Lim C, Azoulay D: Evaluation of the current guidelines for resection of hepatocellular carcinoma using the Appraisal of Guidelines for Research and Evaluation II instrument. Journal of hepatology 2017, 67(5):991-998.

6. Liang L, Safi J, Gagliardi A, Group m: Number and type of guideline implementation tools varies by guideline, clinical condition, country of origin, and type of developer organization: content analysis of 
guidelines. Implementation Science 2017, 12:136.

7. Developing and Evaluating Communication Strategies to support Informed Decision and practice based on Evidence (DECIDE) [http://www.decide-collaboration.eu/]

8. Fearns N, Graham K, Johnston G, Service D: Improving the user experience of patient versions of clinical guidelines: user testing of a Scottish Intercollegiate Guideline Network (SIGN) patient version. BMC Health Serv Res 2016, 16:37.

9. Rosenbaum SE, Moberg J, Glenton C, Schünemann HJ, Lewin S, Akl E, Mustafa RA, Morelli A, Vogel JP, Alonso-Coello $\mathrm{P}$ et al: Developing Evidence to Decision Frameworks and an Interactive Evidence to Decision Tool for Making and Using Decisions and Recommendations in Health Care. Global Challenges 2018, 2(9):1700081.

10. Rosenbaum SE, Glenton C, Cracknell J: User experiences of evidence-based online resources for health professionals: user testing of The Cochrane Library. BMC Med Inform Decis Mak 2008, 8:3434.

11. Rosenbaum S, Glenton C, Nylund H, Oxman A: User testing and stakeholder feedback contributed to the development of understandable and useful Summary of Findings tables for Cochrane reviews. $J$ Clin Epidemiol 2010, 63:607-619.

12. Rosenbaum SE, Glenton C, Oxman AD: Summary-of-findings tables in Cochrane reviews improved understanding and rapid retrieval of key information. Journal of Clinical Epidemiology 2010, 63(6):620-626.

13. Rosenbaum SE, Glenton C, Wiysonge CS, Abalos E, Mignini L, Young T, Althabe F, Ciapponi A, Marti SG, Meng Q et al: Evidence summaries tailored to health policy-makers in low-and middle-income countries. Bull World Health Organ 2011, 89(1):54-61.

14. Liira H, Saarelma O, Callaghan M, Harbour R, Jousimaa J, Kunnamo I, Loudon K, McFarlane E, Treweek S: Patients, health information, and guidelines: A focus-group study. Scandinavian Journal of Primary Health Care 2015, 33(3):212-219.

15. User Experience Model [https://semanticstudios.com/user_experience_design/]

16. Scottish Intercollegiate Guidelines Network: SIGN 145 • Assessment, diagnosis and interventions for autism spectrum disorders. In.; 2016.

17. Scottish Intercollegiate Guidelines Network: A guideline developers' handbook (SIGN CPG 50). In.; 2019.

18. Andrews JC, Schunemann HJ, Oxman AD, Pottie K, Meerpohl JJ, Coello PA, Rind D, Montori VM, Brito JP, Norris $S$ et al: GRADE guidelines: 15 . Going from evidence to recommendation-determinants of a recommendation's direction and strength. J Clin Epidemiol 2013, 66(7):726-735.

19. Balshem H, Helfand M, Schunemann HJ, Oxman AD, Kunz R, Brozek J, Vist GE, Falck-Ytter $Y$, Meerpohl J, Norris S et al: GRADE guidelines: 3. Rating the quality of evidence. J Clin Epidemiol 2011, 64(4):401-406.

20. ASD parent/carer booklet - user testing feedback

[https://www.sign.ac.uk/media/1169/pat145_asd_parent_user_testing_feedback.pdf] 
21. Braun V, Clarke V: Using thematic analysis in psychology. Qualitative Research in Psychology 2006, 3(2):77-101.

22. Lynch PJ HS: Web Style Guide. In., 3rd Edition edn.

23. Autism: A booklet for parents, carers and families of children and young people with autism [https://www.sign.ac.uk/assets/pat145_parents_and_carers.pdf]

24. Santesso N, Morgano GP, Jack SM, Haynes RB, Hill S, Treweek S, Schunemann HJ: Dissemination of Clinical Practice Guidelines: A Content Analysis of Patient Versions. Medical decision making: an international journal of the Society for Medical Decision Making 2016, 36(6):692-702.

25. Oxman AD, Paulsen EJ: Who can you trust? A review of free online sources of "trustworthy" information about treatment effects for patients and the public. BMC Med Inform Decis Mak 2019, 19(1):35.

26. Kristiansen A, Brandt L, Alonso-Coello P, Agoritsas T, Akl EA, Conboy T, Elbarbary M, Ferwana M, Medani W, Murad MH et al: Development of a novel, multilayered presentation format for clinical practice guidelines. Chest 2015, 147(3):754-763.

27. Guideline International Network: The G-I-N PUBLIC Toolkit: Patient and Public Involvement in Guidelines. In.

28. Dreesens D, Stiggelbout A, Agoritsas T, Elwyn G, Flottorp S, Grimshaw J, Kremer L, Santesso N, Stacey D, Treweek $S$ et al: A conceptual framework for patient-directed knowledge tools to support patient-centred care: Results from an evidence-informed consensus meeting. Patient education and counseling 2019, 102(10):1898-1904.

29. van der Weijden T, Dreesens D, Faber MJ, Bos N, Drenthen T, Maas I, Kersten S, Malanda U, van der Scheur S, Post $\mathrm{H}$ et al: Developing quality criteria for patient-directed knowledge tools related to clinical practice guidelines. A development and consensus study. Health Expectations 2019, 22(2):201-208.

30. Wang X, Zhou Q, Chen Y, Yao L, Wang Q, Wang M, Yang K, Norris SL: Protocol of reporting items for public versions of guidelines: the Reporting Tool for Practice Guidelines in Health Care-public versions of guidelines. BMJ Open 2019, 9(3):e023147.

31. Scalia P, Durand M-A, Faber M, Kremer JA, Song J, Elwyn G: User-testing an interactive option grid decision aid for prostate cancer screening: lessons to improve usability. BMJ Open 2019, 9(5):e026748.

32. Ousseine YM, Durand M-A, Bouhnik A-D, Smith $A \bigotimes$, Mancini J: Multiple health literacy dimensions are associated with physicians' efforts to achieve shared decision-making. Patient education and counseling 2019, 102(11):1949-1956.

33. Jansen J, McKinn S, Bonner C, Muscat DM, Doust J, McCaffery K: Shared decision-making about cardiovascular disease medication in older people: a qualitative study of patient experiences in general practice. BMJ Open 2019, 9(3):e026342.

34. Mitter N, Ali A, Scior K: Stigma experienced by families of individuals with intellectual disabilities and autism: A systematic review. Research in Developmental Disabilities 2019, 89:10-21. 
35. Cronin RM, Mayo-Gamble TL, Stimpson S-J, Badawy SM, Crosby LE, Byrd J, Volanakis EJ, Kassim AA, Raphael JL, Murry VM et al: Adapting medical guidelines to be patient-centered using a patientdriven process for individuals with sickle cell disease and their caregivers. BMC Hematology 2018, 18(1):12.

36. Roddis JK, Liversedge HL, Ryder I, Woodhouse M: Incorporating the patient experience into clinical guidelines: recommendations for researchers and guideline developers. BMJ Evidence-Based Medicine 2019, 24(4):125-126.

37. Carroll C: Qualitative evidence synthesis to improve implementation of clinical guidelines. BMJ 2017, 356:j80.

38. Scottish Intercollegiate Guideline Network: Epilepsy in children. In.; in development.

39. Healthcare Improvement Scotland: The experiences of caregivers looking after individuals with Fetal Alcohol Spectrum Disorder: A rapid synthesis of qualitative studies. In.; 2019.

\section{Figures}

Information
Before your child has an autism assessment, professionals making
the referral should do the following.
$\square$ Explain to you that your child's experiences and behaviour
suggest they may have autism
$\square$ Discuss the advantages of further assessment
$\square$ Check your understanding of the reasons for referral
$\square$ Explain to you how long you should expect to wait until your
child goes for assessment
$\square$ Give you a copy of your child's referral letter to read in your
own time
$\square$ Offer you and your family support


Figure 1

information box

Asperger's syndrome is a form of autism where a person has all the main areas of difficulty but does not have learning difficulties or delayed speech.

Figure 2

text box

\section{There are four different types of recommendations in this booklet.}

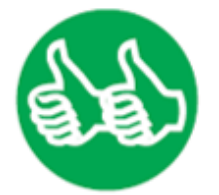

Strong

recommendation

based on good-quality

research evidence.

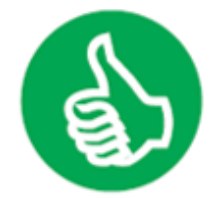

Recommendation based on research evidence.

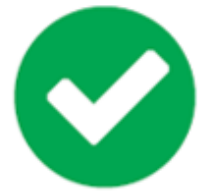

Recommendation based on clinical experience.

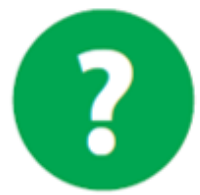

Not enough research evidence to tell us if something is of benefit.

Figure 3

Recommendation levels 


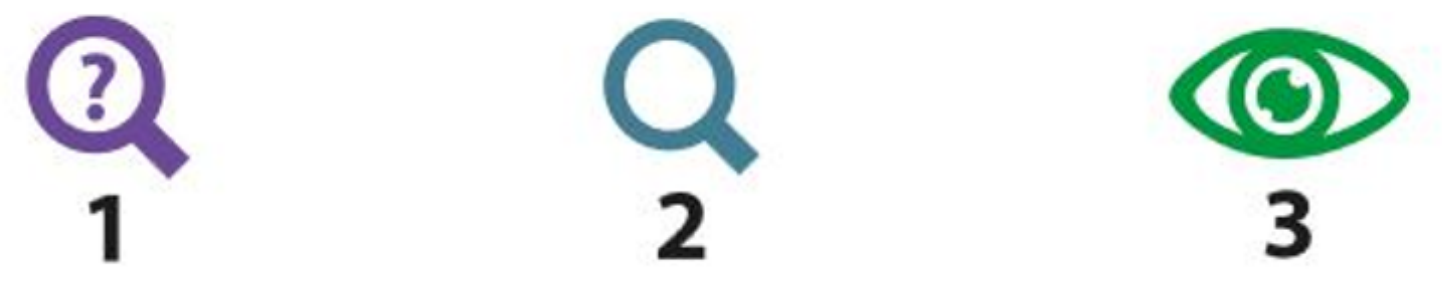

Identify questions Search for evidence Look at the evidence

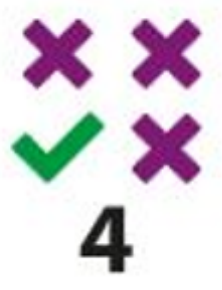

Make judgements and recommendations

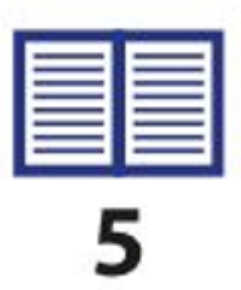

Publish

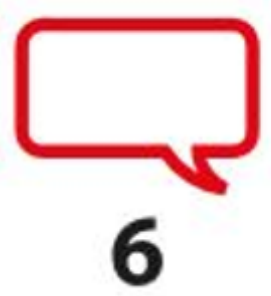

Let everybody know about our guidelines

Figure 4

how SIGN develop their guidelines 